Notre Dame Journal of Formal Logic Volume XVI, Number 2, April 1975

NDJFAM

\title{
A NEW LOOK AT EROTETIC COMMUNICATION
}

\author{
NICHOLAS J. MOUTAFAKIS
}

Recently, attempts have been made to analyse discourse involving questions and answers, and thus to develop its logic. These efforts assume either that questions must be interpreted as propositional functions (with answers being instantiations into these functions), ${ }^{1}$ or questions imply propositions, ${ }^{2}$ or questions are hidden commands. ${ }^{3}$ By adopting one of these views, analysts have sought calculi of interrogatives which work with the precision of propositional logic. The shortcomings of these efforts stem from the very assumption that questions must be reduced to some other "equivalent" grammatical form. In this writers have failed to consider the irreducibility of questions in erotetic communication. Thus their logics have resulted in calculi which do not deal with questions at all, but involve only declaratives of some sort. Moreover, their emphasis upon developing logics which parallel the "true-false" logic of propositions has limited them to the consideration of questions having only "yes" or "no" answers. For they assume that such answers are somehow like the truth values assigned to statements.

This study analyses from a pragmatic viewpoint the unnoticed and multi-faceted nature of discourse concerning questions and answers. Such communication is presented as an interconnected series of sententialized relations between a language user and the language he employs. At every stage it will be shown how key efforts of the past are limited in the way they conceive this communication activity. Significantly, the advantage of seeing responses to questions as distinct assertions of belief sheds new light upon how erotetic communication can be handled without destroying the questions it involves. Finally, some comments will be offered as to how a logic of questions can be developed which does not compromise the role of interrogatives, and which avoids all use of modal operators. In the latter case, the logic of questions will be sketched on the basis of a logic of physical events.

1. [2], p. 353 .

2. [5], p. 30 .

3. [1], p. 142 . 
1 Questions assert nothing which can be true or false. Their legitimate (grammatical) purpose is to produce information so that the questioner can cope with some specifiable situation. Thus answers trigger responses of some productive and therefore determinable sort from the interrogator. If this were not the case, the utterance of a question would be pointless. Keeping in mind this primarily practical function of questions, the activity of questioning can be given an objective characterization. For 'asking' is the indication of an informational hinderance, preventing some activity on the part of the questioner. Here the Greek meaning of 'asking' is helpful. For it is rendered as the aporetic state, meaning the condition of impenetrability due to a lack of pores." Thus the state of "aporia," that is the questioning state, is just that of being unable to go on because of some informational blockage. The notion of 'asking' hence implies no factors which cannot be fully specified. Clearly, a question must indicate that there is some communicable point about which the interrogator lacks certainty, and that this point prevents him from coping with his area of involvement. Whether a question expresses interest in some state of the world, e.g. "Is the chair black?" or in the nature of an activity, e.g., "How does one generate the series: $1,3,5,7, \ldots$ ?' there are elements all questions manifest. For it can be said that they deal with objects, properties, actions, and segments of time.

Thus the symbols ' $X$ ', ' $f$ ', ' $\boldsymbol{a}$ ' and ' $t$ ' are taken as denoting the fundamental elements any question involves, namely an object $X$, a property $f$, an action $a$, and a time-span $t$, during which something is to occur. Though other ingredients may be involved by a question, the ones mentioned seem fundamental. However, whichever of the above elements a question may involve, no question declares the (factual) interassociation of these elements. Rather, object, property, action, and time-span are brought together in questions in the sense of uncertainty (aporia) discussed above. Hence the relation between questioner and question is pragmatico-semantical. For it holds between a language user and the expression of his doubt about the truth of the association of things referred to by the question.

The relation to be sententialized first is trivially that of asking. Hence,

$$
\text { 'I Que }{ }^{t} R \text {, '?Xft }{ }_{1+} \text { ', }
$$

In (1) one has the formula expressing the relation of interrogator $I$ questioning at time $t$ respondent $R$ that 'object $X$ has property $f$ at time $t_{1+}$ ' Thus in (1) the language user is related to the extension of his question by the latter's expression of uncertainty to the respondent as to whether object $X$ has property $f$ at $t_{1+}$. Surely questions of another sort can be introduced in place of the one in (1). For example, interrogator $I$ can question $R$ as to how the series: $1,3,5,7, \ldots$ is to be continued. In essence $I$ is here requesting information as to the kind of action, $a$, which will make series: $1,3,5,7, \ldots, S$, continuous, c, at time $t_{1+}$. Interrogator $I$ and this question can be related as:

4. [3], p. 2978. 


$$
\text { 'I Que }{ }^{t} R, \text { '? } \boldsymbol{a}, S_{\mathrm{ct}}{ }_{1+} \text { ', . }
$$

By (1') one expresses the relation between interrogator $I$ questioning $R$ at $t$ as to what 'action $a$ will make series $S$ continuous c at time $t_{1+}$.' As in (1), there is expressed in ( $\left.1^{\prime}\right)$ the interrogator's doubt concerning the nature of something referred to in the question. However, whereas (1) expresses doubt concerning the association of object and property at some specific time, in (1') doubt is expressed about the action bringing about the object (the series) referred to in the question. The relation of 'questioning' here is again pragmatico-semantical, since it holds between a language user and the extension of his uttered question. For it is the identification of the action, i.e., the specification of its extension, which constitutes the point of the question in (1').

For economy, the symbol '--?--' will be employed to express all questions, whatever their topic of request. Here the advantage of the pragmatic approach already emerges in the way it provides for the simple presentation of a variety of questions. For consideration is not given only to questions requiring assent or denial from the respondent. In contrast, the typical approach has been to consider only questions which can be given "yes" or "no" answers. Harrah's work is exemplary in this respect. In [5] he states that he will consider questions only as statements expressing the questioner's knowledge concerning the "given subject matter.", Hence as statements such questions are said to be true or false, depending upon whether the respondent accepts or challenges by a yes or no answer the expression of knowledge given through the question itself. Similarly, Felix Cohen sees questions as propositional functions, which become true upon a "true" instantiation in these propositional functions. ${ }^{7}$ Thus he also devotes his analysis to questions having only affirmative or negative responses. The questioning of a respondent in itself does not mean that the question intimated will be answered. There is clearly a phase of 'electing' to answer, wherein the respondent consents to answer the uttered question. Here one has a pragmatic relation involving the respondent and the question he elects to answer. It is a relation arising from the respondent recognizing that he can answer the question, i.e., because the question does not violate his intellectual ability, personal well-being, etc. Whatever reason the

5. A greater variety of questions can be expressed than those given in (1) and (1'). For example, where a question deals with whether a certain property is possessed by an object, it can be expressed as: '? $f, X t$ ', meaning what property, $f$, is attributable to object $X$ at time $t$. Again, the suggested approach allows for the expression of complex disjunctive or conjunctive questions such as: '(? $X f t) \vee$ $(? X g t)$ ' and ' $\left(? X f t_{1}\right) \cdot\left(? X g t_{2}\right)$ '. In the former case one has the disjunctive question as to whether object $X$ has property $f$ at time $t$ or whether $X$ has property $g$ at $t$. In the latter case one questions if $X$ has property $f$ at time $t_{1}$ and property $g$ at time $t_{2}$. Thus, the range of the kinds of questions to be symbolized in the above way is limitless.

6. [5], p. 28.

7. [2], pp. 354-355. 
respondent employs for electing to answer the question, it is always specifiable. Thus the next pragmatic relation is that of electing to answer a question. Hence,

$$
\text { ' } R \text { Elec } t_{2} \text { '---?--', Que } I, t \text { '. }
$$

(2) expresses the relation of respondent $R$ electing at time $t_{2}$ question '--?--' as questioned by $I$ at time $t$. Unlike the relation of questioning in (1) and (1'), in (2) one has the relation of a language user to a linguistic inscription, namely the uttered question. Thus (2) is a pragmatico-syntactical relation insofar as the respondent $R$ is saying that '--?--' is an answerable question. Should the respondent find I's question unanswerable, as in the case of a question which requests how to round a square so as to have a round square, he would 'reject' the question. As in (2), there is here a pragmatico-syntactical relation between a language user and the uttered question considered inscriptionally. Here as well it can be objectively specified why the respondent rejects the question. Thus where 'Rei' is used to symbolize the relation of rejection, one has:

$$
\text { ' } R \operatorname{Rej}^{t_{2}} \text { '--?--', Que } I, t \text { '. }
$$

In (3) one has the respondent $R$ rejecting at time $t_{2}$ question '---?--' as questioned by $I$ at time $t .{ }^{8}$

The formulas expressed in (1) and (2) allow for the definition of what counts as the conveying of a question. For employing the symbol 'Con' to express the idea of 'conveying' to a respondent some question '--?--', the following definition emerges:

(D1) $\left(\left(I\right.\right.$ Que $^{t} R$, '--?--') $)\left(R\right.$ Elec $^{t_{2}}$ '--?--', Que $\left.\left.I, t\right)\right) \supset\left(I \operatorname{Con}^{t}{ }^{+} R\right.$, '-- ?--')

(D1) defines conveying a question in terms of the conditional where if $I$ questions at time $t$ respondent $R$ by means of question '--?--' and $R$ elects to answer at time $t_{2}$ question '--?--' as asked by $I$ at time $t$, then interrogator $I$ has conveyed at time $t_{2+}$ to $R$ question '--?--'. The notion of conveying a question introduces an interesting facet in the analysis of the communication activity involving questions. For it is now clear that a question which is responded to is something more than just an uttered interrogative. If one is to retain unaltered the question as it is conveyed, then consideration must be given to the respondent's activity of electing to answer it. This means that a question becomes a functional element in erotetic discourse when it is considered answerable by the respondent.

Most writers analysing questions have thought it proper to see them as statements, which are either prefixed by an interrogative word: "Did you take the money?" or they are declarative statements suffixed by a question mark. The latter are usually given as: "John read the book?" or "The state is just?" From this, analyses proceed to conceive responses to

8. It should be clear that the locution "the questioning of the question" means that the question in point has been presented to the questioner in the sense of (1) and (1'). 
questions as expressions of assent or denial, which constitute accepting as true or false the statement supposedly contained within the question. Here Harrah's summary of major contributors, e.g., Hamblin, Polyany, Leonard, and Miller, is illustrative. For Harrah points out that he, as well as all these writers, grant that the logic of questions already exists within the logic of propositions. However, he goes on to say that in order to secure a logic of questions one must see how they function in the communication activity, which is "... a game played with statements-specifically, an information-matching game." Questions thus become expressions of what the questioner knows about the subject matter, e.g., "'This or that is the case.(?)' or 'Some things are so and so.(?)"' Answers to questions become counter statements which either support, contradict, or augment the information given by the questioner through the question. ${ }^{9}$ Though Harrah attempts to see questions within actual communication, he never gets away from considering them as isolated linguistic entities. Basically, he sees interrogatives as essentially declaratives concerning the interrogator's own knowledge of the subject matter of his question. In view of the pragmatic analysis given above, Harrah's presentation provides no indication as to whether or not the question has been conveyed, that is, whether it has been elected for a response by the respondent. Moreover, if questions are to be viewed as statements of what is "known" by the questioner, then their grammatical purpose of illiciting information is obscured. For as statements questions must be true or false. Yet as questions they assert nothing, and thus cannot be true or false. Thus Harrah presents a view of questions which is ambiguous. Grammatically, his thesis that questions can function both as interrogatives and declaratives is untenable. Harrah attempts to avoid this ambiguity by claiming that questions imply statements concerning what the interrogator knows. ${ }^{10}$ However, what is the sense of implication here? If this is material implication, then the question must be some sort of statement which is implying what the interrogator knows. Thus again he has the question being an expression of uncertainty as to some subject matter, as well as a declarative implying something. Harrah seeks to clarify his case by saying that what animates the vocalization of a question is a psychological intuition about how something should be. ${ }^{11}$ Surely with the introduction of private mental states, and that questions somehow refer to them, one gets into the deeper waters of the possibility of private language, which in no way helps Harrah's conception of questions.

If the response to a question is not the acceptance of some statement supposedly implicit within the question, what is it? How are responses to questions to be handled within a pragmatic analysis? Simply, an answer to

9. [4], pp. 40-41.

10. [5], p. 30 .

11. [5], p. 30. (It is important to observe that Nuel D. Belnap, Jr., in "An Analysis of Questions," Technical Memorandum, 1287, Systems Development Corporation (1963), accepts Harrah's conceptualization of questions, and develops his own logic of questions upon it. (p. 8.)) 
a question is the respondent's statement of "belief" concerning the subject matter of the conveyed question. The extensional pragmatics of belief has been fully developed by R. M. Martin. However, what has not been considered before is that belief statements, insofar as they can be sententialized as extensional and pragmatic relations, constitute the nature of responses to questions. In light of the pragmatic rendition of 'questioning' presented above, it is consistent to conceive the response to a question as an assertion of belief on the part of the respondent concerning the point of information requested by the interrogator. Responses thus become statements which are believed to be true, and they are thus distinct from the questions they are responses for. At this point it becomes clear that the respondent must be the source of authority in erotetic communication, since it makes no sense to ask someone a question without assuming that the person addressed is somehow the source of correct information.

The sententialization of the answer to a question can be presented as:

$$
\text { ' } R \mathrm{Blv}^{t_{3}} \text { 'Xft } \mathrm{H}_{1+} \text { ', }
$$

In (4) one has the expression of the respondent $R$ believing (and uttering) as true at time $t_{3}$ that 'object $X$ has property $f$ at time $t_{1+}$ ' Since the relation expressed in (4) is between respondent and a statement believed by him to be true, (4) expresses a pragmatico-semantical relation.

Also where a response to a question requires the identification of some action, property, etc. it can be rendered as:

$$
\text { ' } R \text { Blv't ' } a, S c t_{1+} \text { ', }
$$

Again in (4') one has a pragmatico-semantical relation between respondent and his believing (and uttering) as true at time $t_{3}$ that action $a$ will make series $S$ continuous c at time $t_{1+}$. Though the responses expressed in (4) and $\left(4^{\prime}\right)$ deal with actions, objects, properties, and time-spans referred to in the questions expressed by (1) and (1') respectively, there is nothing in this conceptualization of responses which suggests that there is an implicit assertion which is contained within the question and expressed in the response. Rather, the relations expressed by (4) and (4') stand alone as expressions of the respondent's assertion that such and such is true. Thus it is absurd to think that the interrogator's question can in any way contain the respondent's belief statement.

Where an answer to a question is given, the interrogator 'accepts' the answer, meaning that he is willing to act upon what the response says so as to alleviate the informational blockage he has encountered. Thus where the symbol ' $--r--$ ' is used to express responses generally, the questioner's acceptance of the response receives the following formulation:

$$
\text { 'I Acpt }{ }^{t_{4}} \text { '- }-r-- \text { ', Blv } R, t_{3} \text { '. }
$$

Thus in (5) interrogator $I$ accepts at time $t_{4}$ response '-- $r--$ ' as believed by respondent $R$ at time $t_{3}$. What the interrogator is related to in (5) is the response as an inscription, which serves to direct the interrogator to act in such and such a way so as to circumvent the impasse he is confronted with. 
Thus 'acceptance' is a pragmatico-syntactical relation. In light of the presentation of the interrogator accepting the response, one must reconsider Lennart Aqvist's contention that questions should be handled as imperatives which demand of the respondent that the latter "... widen... the questioner's knowledge." Here questions are taken to be synonymous with imperative expressions requiring the receiver "let it (turn out to) be" that the questioner knows this or that is the case. ${ }^{12}$ As accepted, a response is an assertion of belief which directs the questioner to act in a way which will remove the impasse which gave rise to the question. However, if a question is taken as an imperative, as Aqvist claims, then it is hard to see how meaningful question-answer communication can go on if both sides are giving each other commands. Furthermore, how can a response to a question make it the case that the interrogator "knows" something? Aqvist only says that the knowledge derived from the response is determined by whether or not the response satisfies the desire which initiates the question. ${ }^{13}$ He never explains how this satisfaction is to be understood. The difficulty here stems from his explicit denial that an answer to a question has a primarily pragmatic function which enables the interrogator to resolve his difficulty. ${ }^{14}$ Hence the role of the response in Aqvist's analysis acquires a vague emotional import, which hampers the understanding of his conception of question-answer communication.

It is hard to see how correct answers to questions can be conceived in any other way than as directives of practical import. For when the questioner finds that the belief assertion he has accepted works in alleviating the difficulty which gave rise to the question, he knows he has a "correct answer." It is important to note how the present analysis of erotetic communication allows for a sensitive distinction between the response itself as a statement of belief, and the response as a correct response for the interrogator. The latter constitutes the last phase in the pragmatic sententialization of question-answer discourse. Hence, where 'Prf' expresses the questioner's preference for that response which functions usefully, the following formula emerges:

$$
\text { ' } I \text { Prf } t_{5} \text { ' }--r-- \text { ', Blv } R t_{3} \cdot \text { Acpt } I t_{4} \text { '. }
$$

Formula (6) expresses interrogator $I^{\prime}$ 's preference at time $t_{5}$ for response '--r--' as believed by respondent $R$ at time $t_{3}$ and accepted by $I$ at time $t_{4}$. In essence the notion of preference is the relation where the interrogator actually finds that the statement believed true by the respondent has a correspondence with the way things are in the area of concern, so that action upon the response yields productive activity. Hence there is nothing other than the observable effect of the response which can be pointed to as the criterion for the correctness of the response. For example, in (4'), if the action referred to by the respondent does generate the series in a recognizably consistent manner, then that response is preferred by the

12. [1], p. 142 .

13. [1], p. 144.

14. [1], p. 145. 
interrogator. Thus the relation of preference is pragmatico-semantical in that it holds between the interrogator and the response as a "correct" response. The advantage of conceiving correct responses to questions as preferred responses lies in the twofold flexibility of the notion of preference in a question-answer context. For in a simple sense it can be said that the correct response is preferred to the situation of being unable to cope with the question's topic of concern. Secondly, by conceiving correct responses as preferred responses, one provides for the case where the questioner selects from a variety of responses the one which is most productive.

Finally, the conjunction of the notions of conveying a question and preferring a response secures the definition of erotetic communication from a pragmatic viewpoint. Hence the last definition to be offered defines the symbol for erotetic communication, ' $\mathrm{ErD}$ ', as follows:

$$
\begin{aligned}
& \left(\left(I \operatorname{Con}^{t_{2}+} R,,^{6}--?--'\right) \cdot\left(I \operatorname{Prf}^{t_{5}}--r--', \text { Blv } R t_{3} \cdot \operatorname{Acpt} I t_{4}\right)\right) \supset \\
& \text { (I } \mathrm{ErD}^{\left.t-t_{5} R,{ }^{6}--?--'\right)} \text {. }
\end{aligned}
$$

Simply, definition (D2) says that if questioner $I$ conveys at time $t_{2}$ to respondent $R$ question '--?--' and $I$ prefers at time $t_{5}$ response '--r--' as believed by $R$ at $t_{3}$ and accepted by $I$ at $t_{4}$, then $I$ is erotetically discoursing, ErD, during time-span $t$ to $t_{5}$ with $R$ about question '--?--'.

Throughout this analysis of the pragmatic topology of erotetic discourse emphasis has been placed upon presenting the fundamental and objectively determinable relations constituting question-answer discourse. As a result nowhere in the explication of 'questioning,' 'electing,' 'believing,' 'accepting,' and 'preferring' is there reliance upon connotative meanings so as to clarify any of these relations. Each phase of question-answer discourse has been articulated as a relation between a language user and an expression, so that one outcome has been the possibility of treating an unlimited variety of questions. Moreover, nowhere in this analysis has the role of the question in discourse been compromised, so as to have it reinterpreted into a declarative or imperative. In this respect the analysis has preserved the natural character of erotetic discourse while also unearthing its unnoticed complexity.

2 If questions are not statements, how is their logic to be articulated? What other model can be used to present an erotetic logic than two-valued propositional logic? Though this study does not aim at developing a logic of questions, it does seek to suggest how such a logic can be had by considering some new developments in the area of praxiology. The first step will be to consider the communication event of erotetic discourse as a physical event, having all of its essential elements specifiable within a physicalistic object language. This should not be difficult since in the previous section erotetic discourse was found to be open to a characterization involving only pragmatic relations. Secondly, the formal interrelations of such events shall be presented as derived from R. M. Martin's theory of events. ${ }^{15}$ Thus

15. [7], Chapter IX. 
a logic of questions and answers will be proposed which dispenses with basing it upon a logic of propositions, and which avoids all use of modal operators.

Erotetic communication can be characterized as a relational event involving an interrogator, a respondent, the pragmatic relation of erotetic discourse, and a time-span during which the question-answer communication occurs. Thus one can present as a virtual ordered quadruple the event predicate of an erotetic event as: ' $\langle I, \operatorname{ErD}, R$, ' -- ?--', $t>$ '. The symbolization of an elemental erotetic event is thus:

$$
\text { '<I, ErD, } R \text {, '--?--', } t>\boldsymbol{e}^{\prime} \text {. }
$$

Thus in (i) where $e$ is a variable ranging over events, then (i) expresses the event of interrogator- $I$-erotetically-discoursing-with-respondent- $R$-concerning-question-'--?--'-during-time-span- $t$. The event expressed in (i) provides new depth to the meaning of the simple expression of an interrogative. The definition which follows illustrates this point.

$$
\begin{aligned}
& \text { '--?--' }{ }_{D f}(\exists I)(\exists R)(\exists t)\left(\exists^{6}-- \text { ?--' }\right)\left(I \mathrm{ErD}{ }^{t} R,\right. \text { '--?--' . } \\
& \left.<I, \operatorname{ErD}, R, t,{ }^{\prime}--?--’>\hat{e}\right) \text {. }
\end{aligned}
$$

The interrogative '--?--' above is seen to involve a number of objects. Hence its definition is presented as saying that there is an interrogator $I$, respondent $R$, time $t$, and question '--?--', such that $I$ discourses erotetically with $R$ during $t$ concerning '--?--' and $I$-discoursingerotetically-at-t-about-'--?--' is an event $\boldsymbol{e}$.

Having an objective characterization of an erotetic event, hereafter symbolized as '? $e$ ' one can proceed to give the logic of such events as an extension of the logic of physical events. The latter contains the elemental relations of beforeness, overlapping, local temporal part, local simultaneity, and distinctness. Briefly, the above five relationships will be interpreted within the framework of events involving questions and answers. Hence, where "beforeness" is defined as:

Def. I. " $e_{1} \mathrm{~B} e_{2}$ ' expresses that $e_{1}$ is before $e_{2}$ in the Eigenzeit or local time of the observer.,"16

Definition I. can be expressed as the dependence of one erotetic event upon another. For if one question cannot be answered before another, then the event involving the former question must be manifest after the event containing the latter question. In view of this one can postulate the following:

P1. $\left(e_{1}\right)\left(e_{2}\right)\left(\left(? e_{1} \cdot ? e_{2}\right) \supset\left(\left(e_{2} \operatorname{Dep} e_{1}\right) \supset\left(e_{1} \mathrm{~B} e_{2}\right)\right)\right)$.

Postulate P1. says that for every event $\boldsymbol{e}_{1}$ and every event $\boldsymbol{e}_{2}$, if $\boldsymbol{e}_{1}$ and $\boldsymbol{e}_{2}$ are both erotetic events, then that $e_{2}$ is dependent upon ('Dep') $e_{1}$ implies that $e_{1}$ comes before event $e_{2}$. The above postulate would cover the case where one has the following two questions: "Have you read the book?" and "How does the author conclude the book?"' Clearly, it would make no sense

16. [6], p. 9 . 
to ask the latter question if one does not have a response to the first, relative to the same respondent, book, and time period in both questions.

Continuing, where the symmetrical relation of two events "overlapping" is defined as:

Def. II. "Event $e_{1}$ may ... be said to locally overlap temporally,... , with event $e_{2}$ provided neither bears $B$-relation to the other. [Hence...] ' $e_{1}$ LTO $e_{2}$ ' abbreviates: '( $\left.\sim e_{1} B e_{2} \cdot \sim e_{2} B e_{1}\right)$ '.",

this is comparable to the nondependence of one event upon another. For if two erotetic events can be answered without the one necessarily preceding the other, then neither erotetic event is dependent upon the other. This relation can be postulated as:

P2. $\left(e_{1}\right)\left(e_{2}\right)\left(\left(? e_{1} \cdot ? e_{2}\right) \supset\left(\left(\sim e_{1} \operatorname{Dep} e_{2}\right) \cdot\left(\sim e_{2} \operatorname{Dep} e_{1}\right)\right) \supset\left(\left(\sim e_{1} B e_{2}\right) \cdot\left(\sim e_{2} B e_{1}\right)\right)\right)$.

P2 postulates that where $e_{1}$ and $e_{2}$ are both erotetic events, then if $e_{1}$ is not dependent upon $\boldsymbol{e}_{2}$ and $\boldsymbol{e}_{2}$ is not dependent upon $\boldsymbol{e}_{1}$, then $\boldsymbol{e}_{1}$ is not before $e_{2}$ and $e_{2}$ is not before $e_{1}$. The nondependence of erotetic events is involved in cases where some questions can be answered while other questions are being answered as well. Hence, given the two questions: "What are the ways by which geologic change is possible?" and "When did man first appear on this planet?" each question can be answered prior to the other. For the first question does not have temporal priority over the second, since geologic change has been continuing since man's presence. On the other hand, man's presence on earth in no way antecedes geologic change, nor does it govern such change.

The definition of the asymmetrical relation of "local temporal part" emerges from that of local temporal overlapping as follows:

Def. III. ". . . event $\boldsymbol{e}_{1}$ is a local temporal part of event $\boldsymbol{e}_{2}$ provided every event which overlaps temporally with $e_{1}$ overlaps also with $e_{2}$. ' $e_{1}$ LTP $e_{2}$ ' abbreviates ' $(e)\left(e\right.$ LTO $e_{1} \supset e$ LTO $\left.e_{2}\right)$ '.,"18

Again, within the framework of question-answer discourse, the notion of "local temporal part" is involved in cases where one event is found to response contain another event. In this case the two erotetic events are such that an answer to the former will constitute an answer to the latter as well. For example, given the two questions: "Which are the capitals of the countries in Europe?" and "What is the capital of France?" an answer to the latter does not constitute an answer to the former question. However, an answer to the first question involves an answer to the second. Here it can be said that the second is "response contained" by the first question. The formal postulation of response containment for erotetic events can be set forth as:

P3. $\left(e_{1}\right)\left(e_{2}\right)\left(\left(? e_{1} \cdot ? e_{2}\right) \supset\left(\left(e_{1} \operatorname{Res} C o n e_{2}\right) \supset\left((e)\left(e\right.\right.\right.\right.$ LTO $e_{1} \supset e$ LTO $\left.\left.\left.\left.e_{2}\right)\right)\right)\right)$.

The above postulate states that if events $e_{1}$ and $e_{2}$ are both erotetic events,

17. $[6]$, p. 9 .

18. [6], p. 9 . 
then $e_{1}$ response contains $e_{2}$ if for every event $e, e$ is a local temporal part of $e_{1}$ implies that $e$ is a local temporal part of $e_{2}$.

The two remaining relations, local simultaneity and discreteness, again have their application within erotetic discourse. Thus the symmetrical relation of "local simultaneity" is defined by R. M. Martin as follows:

Def. IV. "An event $\boldsymbol{e}_{1}$ is locally simultaneous with event $\boldsymbol{e}_{2}$ provided every local temporal part of $e_{1}$ is part of $e_{2}$, and conversely. Thus ' $e_{1}$ Simul $e_{2}$ ' abbreviates ' $(e)\left(e \operatorname{LTP} e_{1} \equiv e \operatorname{LTP} e_{2}\right)$ '.,"19

With respect to talk about questions, the relation presented in Def. IV. is manifest in cases where one response can satisfy two distinct questions. A case in point is where one has the questions: "Have you seen our largest city?" and "Did you get to New York City?" Relative to the same respondent and time period, one response can satisfy both of the above questions, assuming that he who answers knows that New York is the largest city in the country.

The formal postulation of satisfaction equivalence can be presented in the following way:

P4. $\left(e_{1}\right)\left(e_{2}\right)\left(\left(? e_{1} \cdot ? e_{2}\right) \supset\left(\left(e_{1}\right.\right.\right.$ Sat.Equi. $\left.\left.\left.e_{2}\right) \supset\left((e)\left(e \operatorname{LTP} e_{1} \equiv e \operatorname{LTP} e_{2}\right)\right)\right)\right)$.

Postulate P4. states that for every event $\boldsymbol{e}_{1}$ and $\boldsymbol{e}_{2}$, where $\boldsymbol{e}_{1}$ and $\boldsymbol{e}_{2}$ are both erotetic events, then $e_{1}$ is satisfiably equivalent to $e_{2}$ if for every event $e$, that $e$ is a local temporal part of $e_{1}$ is equivalent to $e$ being the local temporal part of $\boldsymbol{e}_{2}$.

Finally, "discreteness" is definable as the following symmetrical relation:

Def. V. ". . . the event $e_{1}$ is discrete from $e_{2}$ provided they occur at different times or in different places. . . , 20

This relation can be rendered as the total independence of one erotetic event from another. Thus where two erotetic events do not exhibit any of the four preceding relations, then these two events are independent of each other. Employing Martin's symbol for discreteness, '/, one can postulate this relation as follows:

P5: $\left(e_{1}\right)\left(e_{2}\right)\left(\left(? e_{1} \cdot ? e_{2}\right) \supset\left(\left(e_{1}\right.\right.\right.$ indep $\left.\left.\left.e_{2}\right) \supset\left(e_{1} / e_{2}\right)\right)\right)$.

P5. reads that for every event $e_{1}$ and $e_{2}$, if these are both erotetic events, then $e_{1}$ is independent of $e_{2}$ if $e_{1}$ is discrete from $e_{2}$.

Much can be said as to how the above relations yield events of greater complexity. However, space forbids further comment. All that can be noted in the proposed logic of questions is the absence of any modal operators, such as "ought," "permissible," "possible," etc. Rather, each of the five postulates given above have been articulated on the basis of clear and precise relationships holding between physical events, together with some basic notions from formal logic.

19. [6], p. 9 .

20. [6], pp. 7 and 8 . 
Both in the pragmatic analysis of erotetic discourse, and in the sketching of the logic of erotetic events, a new approach has been indicated. To the time of this writing, the pragmatics of questions has not been considered with any of the above detail. Furthermore, the innovation of looking at responses to questions pragmatically as assertions of belief promises a new direction in the analysis of this area of communication, one which does not compromise the role of the interrogative. Finally, the proposed logic of erotetic events appears simpler and less complex than the so-called modal logics of questions.

\section{REFERENCES}

[1] Åqvist, L., A New Approach to the Logical Theory of Interrogatives, Part I, Uppsala (1965).

[2] Cohen, F., "What is a question?," Monist, vol. 39 (1929), pp. 350-364.

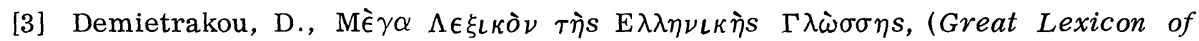
the Hellenic Language), Athens (1938).

[4] Harrah, D., "A logic of questions and answers," Philosophy of Science, vol. 28 (1961), pp. 40-46.

[5] Harrah, D., Communication: A Logical Model, M.I.T. Press (1963).

[6] Martin, R. M., Events, Histories, Physical Objects: Some Logico-Metaphysical Reflections (1968), mimeograph copy.

[7] Martin, R. M., Belief, Existence, and Meaning, New York University Press (1969).

The Cleveland State University

Cleveland, Ohio 